\title{
Fast segmentation of the Left Atrial Appendage in 3D Transesophageal Echocardiographic images
}

\author{
Pedro Morais, Sandro Queirós, Pieter De Meester, Werner Budts, João L. Vilaça, João Manuel R. S. Tavares, \\ Jan D'hooge
}

\begin{abstract}
Left atrial appendage (LAA) has been generally described as "our most lethal attachment", being considered the major source of thromboembolism in patients with non-valvular atrial fibrillation. Currently, LAA occlusion can be offered as a treatment for these patients, obstructing the LAA through a percutaneously delivered device. Nevertheless, correct device sizing is not straightforward, requiring the manual analysis of peri-procedural images. This approach is sub-optimal, time demanding and highly variable between experts, which can result in lengthy procedures and excess manipulations. In this article, a semi-automatic LAA segmentation technique for 3D transesophageal echocardiography (TEE) images is presented. Specifically, the proposed technique relies on a novel segmentation pipeline where a curvilinear blind-ended model is optimized through a double stage strategy: 1) fast contour evolution using global terms and 2) contour refinement based on regional energies. To reduce its computational cost, and thus make it more attractive to real interventions, the B-spline Explicit Active Surface framework was used. This novel method was evaluated in a clinical database of 20 patients. Manual analysis performed by two observers was used as ground truth. The 3D segmentation results corroborated the accuracy, robustness to the variation of the parameters and computationally attractiveness of the proposed method, taking approximately 14 seconds to segment
\end{abstract}

This work was funded by projects NORTE-01-0145-FEDER-000013, NORTE-01-0145-FEDER-000022 and NORTE-01-0145-FEDER-024300, supported by Northern Portugal Regional Operational Programme (Norte2020), under the Portugal 2020 Partnership Agreement, through the European Regional Development Fund (FEDER), and also been funded by FEDER funds, through Competitiveness Factors Operational Programme (COMPETE), and by national funds, through the FCT - Fundação para a Ciência e Tecnologia, under the scope of the project POCI-01-0145FEDER-007038. The authors acknowledge support by FCT and the European Social Found, through Programa Operacional Capital Humano (POCH), in the scope of the $\mathrm{PhD}$ grants SFRH/BD/95438/2013 (P. Morais) and SFRH/BD/93443/2013 (S. Queirós). Asterisk indicates corresponding author.

*P. Morais is with the Lab on Cardiovascular Imaging and Dynamics, KU Leuven, Belgium, with Life and Health Sciences Research Institute (ICVS), School of Medicine, University of Minho, 4710-057, Braga, Portugal, with ICVS/3B's - PT Government Associate Laboratory, Braga/Guimares, Portugal, Instituto de Ciência e Inovação em Engenharia Mecânica e Engenharia Industrial, Departamento de Engenharia Mecânica, Faculdade de Engenharia, Universidade do Porto, Porto, Portugal and 2Ai - Polytechnic Institute of Cávado and Ave, Barcelos, Portugal (e-mail: pedromorais@med.uminho.pt).

S. Queirós is with Life and Health Sciences Research Institute, ICVS/3B's - PT Government Associate Laboratory, Algoritmi Center, School of Engineering, University of Minho, Portugal, and Lab on Cardiovascular Imaging and Dynamics, KU Leuven, Belgium.

P. Meester and W. Budts are with Department of Cardiology, University Hospitals Leuven, Leuven, Belgium.

J. L. Vilaça is with $2 \mathrm{Ai}$ - Polytechnic Institute of Cávado and Ave, Barcelos, with Life and Health Sciences Research Institute and ICVS/3B's PT Government Associate Laboratory, University of Minho, Portugal.

J. M. R. S. Tavares is with Instituto de Ciência e Inovação em Engenharia Mecânica e Engenharia Industrial, Departamento de Engenharia Mecânica, Faculdade de Engenharia, Universidade do Porto, Porto, Portugal.

$\mathrm{J}$. D'hooge is with the Lab on Cardiovascular Imaging and Dynamics, KU Leuven, Belgium. the LAA with an average accuracy of $\sim 0.9 \mathrm{~mm}$. Moreover, a performance comparable to the inter-observer variability was found. Finally, the advantages of the segmented model were evaluated while semi-automatically extracting the clinical measurements for device selection, showing a similar accuracy but with a higher reproducibility when compared to the current practice. Overall, the proposed segmentation method shows potential for an improved planning of LAA occlusion, demonstrating its added value for normal clinical practice.

Index Terms-3D image segmentation, Left atrial appendage, curvilinear blind-ended model, B-spline Explicit Active Surface.

\section{INTRODUCTION}

$\mathbf{L}$ eft atrial appendage is the remnant of the embryonic left atrium (LA) and it is commonly described as a long tubular, trabeculated blind-ended structure attached to the LA body (Fig. 1) [1]-[3]. High variability in LAA shape and morphology is expected between subjects and different size and complex multi-lobular structures are common. Although doubts remain about its real function [4], some recent studies proved that the LAA is the source of thromboembolism in approximately $90 \%$ of patients with nonvalvular atrial fibrillation (NVAF) [1], [2]. Anticoagulation therapy is clinically accepted as the main embolism prevention treatment for patients with atrial fibrillation, but with a poor long-term compliance and potential bleeding complications [3], [5]. In this sense, percutaneous LAA occlusion appeared as a recommended treatment for NVAF patients that present high stroke risk and contraindications for oral anticoagulation treatment [2], [6].

The LAA occlusion is a minimally-invasive cardiac intervention, where a specific foldable device is percutaneously delivered, via a transseptal route [7], at the LAA ostium obstructing the orifice and reducing the risk of a thromboembolism [1]. The procedure is guided through fluoroscopy and transesophageal echocardiography (TEE) imaging, requiring a pre-procedural TEE examination to rule out thrombus and to evaluate the LAA morphology. Moreover, recently, researchers [8]-[12] have proven the added-value of computed tomography (CT) acquisition for assessment of the LAA shape.

Due to the high shape variability between subjects, different manufacturers produce occluding devices with different sizes and shapes [1]. As a result, clinical measurements, which vary between manufacturers, must be taken to identify the optimal model, increasing the difficulty of the intervention. Indeed, recent studies suggest that the device selection stage is one of the most critical stages during LAA occlusion [12], [13]. Although some experts prefer to identify the device size based 


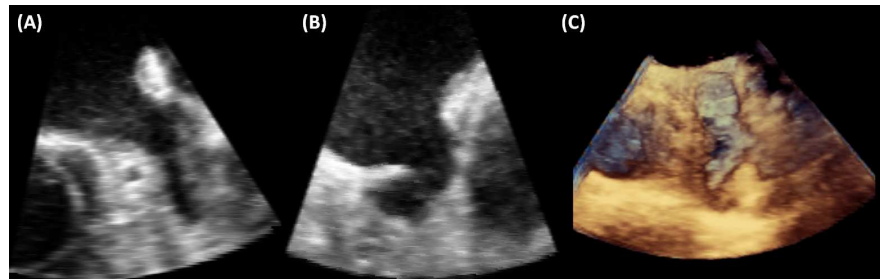

Fig. 1. (A-B) Orthogonal 2D views and (C) 3D representation of the LAA.

on detailed pre-procedural data (i.e., CT) [12], [14], [15], the majority of the interventionists still performs this stage using peri-procedural imaging. Indeed, since the $\mathrm{CT}$ data is acquired before the intervention, anatomical variations can occur, which may influence the selected device [16]. For this reason, during the real intervention, the experts perform multiple image acquisitions (both fluoroscopy and TEE) and visually assess the resulting images to identify the device [17]. However, such approach still presents several disadvantages, namely: 1) it is time-consuming, requiring several minutes to correctly identify the optimal device; 2 ) the correct identification of the 3D LAA shape through peri-procedural data is not straightforward; and 3 ) the clinical analysis is totally manual, lacking intra- and inter-observer reproducibility [8].

Recently, a few studies focusing on novel planning techniques for LAA occlusion were presented [8], [9], [18]. The majority of them rely on 3D-printed models. More specifically, the LAA anatomy is extracted from CT [9] or TEE images [18], being subsequently edited and physically generated through 3D-printers. The accurate model is then provided to the physician prior to the intervention, allowing a correct interpretation of the LAA shape and identification of the optimal occluding device. Although this valuable approach has gained, recently, particular attention, it is complex, time demanding (several hours to print the 3D model) and not automated, requiring user-interaction at multiple stages. Other researchers developed image processing techniques to segment the target anatomy, particularly for CT datasets, using simple imagebased techniques [8], deformable models [19] or machine learning strategies [20], [21]. Interestingly, the image-based technique from Song et al. [8] was also applied in TEE images, extracting realistic LAA shapes but being extremely timedemanding due to the high number of manual corrections required. Indeed, taking into consideration the state-of-theart, efficient and automated strategies to segment the LAA in TEE images were, to the authors' best knowledge, not described, particularly due to: 1) the complex curvilinear and tubular anatomical shape of the LAA; 2) the high anatomical variability of this structure; and 3) the low image quality.

In this article, a novel strategy is proposed to accurately and quickly segment the LAA anatomy in 3D-TEE images, which can ease the interpretation of peri-procedural ultrasound data and ultimately has the potential to simplify LAA occlusion planning. The novel strategy captures all the anatomical particularities of the LAA by applying a double stage segmentation process focused on the evolution of a curvilinear blind-ended model. In detail, a standard model is initially generated by manually defining its centerline, being then refined to the patient anatomy through a fast contour evolution approach with global descriptors followed by a refined image segmentation step with regional terms. The segmentation method was implemented based on the B-spline Explicit Active Surface framework (BEAS), extending its current formulation by presenting a novel curvilinear blind-ended BEAS model.

Hereupon, the current work introduces two novelties: 1) a novel LAA segmentation technique for 3D-TEE images based on the evolution of a curvilinear blind-ended BEAS model; and 2) a clinical validation of the proposed method, by comparing its performance against the traditional manual practice in terms of segmentation accuracy and reliability of the extracted clinical measurements.

The article is structured as follows. In section II, a technical description of the segmentation pipeline is presented. An initial explanation of the curvilinear blind-ended BEAS model is provided, being afterward described the different steps of the proposed LAA segmentation pipeline. In Section III and Section IV, the validation experiments and the results are presented, respectively. Section V evaluates and discusses the performance of the proposed method. Finally, the conclusions of the work are presented in Section VI.

\section{Methods}

In this section, a description of the LAA segmentation pipeline is presented. We start by explaining the basic concepts of the BEAS framework (section II-A), later expanding its formulation to a curvilinear blind-ended model (section II-B), making it suitable to capture the particularities of the LAA anatomy. Finally, a detailed description of the novel doublestage LAA segmentation framework applied to optimize the curvilinear surface is presented (section II-C).

\section{A. B-spline Explicit Active Surfaces}

The key concept of the BEAS framework is to represent an object interface as an explicit function described as a linear combination of B-spline basis functions [22]. Thanks to this explicit description, a reduction of the dimensionality $n$ of the segmentation problem is possible, being one of the coordinates of the interface points, $\mathbf{x}=\left\{x_{1}, x_{2}, \ldots, x_{n}\right\}$, described as a function of the remaining ones, $x_{1}=\psi\left(\mathbf{x}^{*}\right)=\psi\left(x_{2}, \ldots, x_{n}\right)$. In this sense, the explicit function $\psi$ is defined as:

$$
x_{1}=\psi\left(x_{2}, \ldots, x_{n}\right)=\sum_{\mathbf{k} \in \mathbb{Z}^{n-1}} c[\mathbf{k}] \beta^{d}\left(\frac{\mathbf{x}^{*}}{h}-\mathbf{k}\right),
$$

where $\beta^{d}($.$) is the uniform symmetric (n-1)$-dimensional Bspline of degree $d$. The knots of the B-splines are located on a grid defined on a specific coordinate system, with a spacing represented by $h . c[\mathbf{k}]$ represents the B-spline coefficients.

The evolution model is controlled by a regional-based energy $(E)$, defined as:

$$
E=\int_{\Omega} \delta_{\phi}(\mathbf{x}) \int_{\Omega} B(\mathbf{x}, \mathbf{y}) \cdot F(\mathbf{y}) \partial \mathbf{y} \partial \mathbf{x},
$$

where $\mathbf{x}, \mathbf{y}$ represents independent spatial locations in the image domain $\Omega . \delta_{\phi}$ is the Dirac function and $F(\mathbf{y})$ is an image criteria (e.g., local Chan-Vese, [23]). $B(\mathbf{x}, \mathbf{y})$ is a local 
mask function where the regional parameters that drive the contour evolution are estimated and it is defined as a set of points $\mathbf{y}$ along the normal direction $(\hat{\mathbf{r}})$ of $\mathbf{x}$ whose distance is lower than $\rho$ [23]:

$$
B(\mathbf{x}, \mathbf{y})=\left\{\begin{array}{ll}
1, & \text { if } \mathbf{y}=\mathbf{x}+m \times \hat{\mathbf{r}}, m \in[-\rho ; \rho] \\
0, & \text { otherwise }
\end{array} .\right.
$$

The energy minimization is performed using a gradient descent approach through the computation of the energy derivatives with respect to each $\mathrm{B}$-spline coefficient $c[\mathbf{k}]$ :

$$
\begin{gathered}
\frac{\partial E}{\partial c[\mathbf{k}]}=\int_{\Gamma} \bar{g}\left(\mathbf{x}^{*}\right) \beta^{d}\left(\frac{\mathbf{x}^{*}}{h}-\mathbf{k}\right) \partial\left(\mathbf{x}^{*}\right), \\
\bar{g}\left(\mathbf{x}^{*}\right)=\bar{g}^{I M}\left(\mathbf{x}^{*}\right)+\alpha \cdot \bar{g}^{\operatorname{Reg}}\left(\mathbf{x}^{*}\right),
\end{gathered}
$$

$\phi(\mathbf{x})$ is a level-set like function representing the region inside the interface $\Gamma\left(\phi(\mathbf{x})=\Gamma\left(\mathbf{x}^{*}\right)-x_{1}\right)$ and $\bar{g}^{I M}\left(\mathbf{x}^{*}\right)$ is the first derivative of $F(\mathbf{y})$ (e.g. first derivative of the local ChanVese [23]). In order to reduce the sensibility of the model to local minima, a shape-based regularization term $\bar{g}^{R e g}\left(\mathbf{x}^{*}\right)$ (e.g. curvature-based regularization) is included in the final feature map $\bar{g}\left(\mathbf{x}^{*}\right) . \alpha$ is a positive hyperparameter that balances the image-based and regularization terms. It must be specifically defined based on the selected terms (e.g. regularization strategy used). For further details on the BEAS mathematical formalism, the reader is kindly directed to [22].

\section{B. Curvilinear blind-ended model}

1) Description: In order to capture the particularities of the LAA shape, a novel BEAS model is proposed. Hereto, a description of the tubular blind-ended LAA shape is presented as a combination of both cylindrical and spherical spaces. Moreover, in order to take into account the LAA anatomical variability (allowing straight and non-straight shapes), a cylindrical system defined along a pre-defined curved axis is used [24], [25].

Fig. $2 \mathrm{a}$ presents an illustrative schematic of the proposed model. Each coordinate $\mathbf{x}$ of the 3D surface is described in terms of its longitudinal $\left(p_{L}\right)$ and circumferential coordinates $\left(p_{C}\right)$ plus its respective orthogonal distance $(r)$ to the curved centerline axis, i.e., $\mathbf{x}=\left\{r, p_{L}, p_{C}\right\}$. Two independent spaces are used to describe the target model: 1) the tubular body of the LAA is defined based on a cylindrical space with a curved axis (i.e., $p_{L}$ and $p_{C}$ described as the distance $s$ along the axis and azimuth $\theta$, respectively) and the LAA tip through a spherical coordinate system (i.e. $p_{L}$ and $p_{C}$ described as elevation $\varphi$ and azimuth $\theta$, respectively). Considering a long-axis view as presented in Fig. 2a, a set of $p_{L}$ coordinates with different positions and orientations, which were defined based on the model centerline and the tangent to each position, is used to generate the curvilinear tubular surface (green line in Fig. 2a). Then, at the final portion of the cylindrical model, a half sphere is positioned by defining the $p_{L}$ coordinate according to a spherical space (blue line in Fig. 2a), ultimately generating the blind-ended LAA model. In order to describe this model with the BEAS framework, Equation 1 is now rewritten as: with,

$$
r=\psi\left(p_{L}, p_{C}\right)=\psi\left(\mathbf{x}^{*}\right)
$$

$$
\begin{gathered}
p_{L}=\left\{\begin{array}{ll}
s & \text { if } s<s_{\text {Max }} \\
\varphi_{s_{\text {Max }}} & \text { if } s=s_{\text {Max }}
\end{array}\right. \text { and } \\
p_{C}=\theta,
\end{gathered}
$$

where $s_{\text {Max }}$ defines the $s$ level interface between the cylindrical and spherical components. Additionally, $\theta \in[0 ; 2 \pi[$, $s \in\left[0 ; s_{\operatorname{Max}}\left[\right.\right.$ and $\varphi_{s_{\text {Max }}} \in\left[\frac{\pi}{2} ; \pi[\right.$.

Finally, the current 3D model is evolved using a discretized surface grid with size $\left(n_{s}+n_{\varphi}\right) \times n_{n_{\theta}}$. The terms $n_{s}$ and $n_{\varphi}$ express the size of the cylindrical and spherical grid component at the longitudinal direction, respectively. $n_{\theta}$ represents the size of the grid at the circumferential direction. Therefore, each point of the grid is described as $\mathbf{x}(i, m)=$ $\left[r_{i, m}, p_{L_{i}}, p_{C_{m}}\right]$, with $i \in\left[0 ; n_{s}+n_{\varphi}\left[, m \in\left[0 ; n_{p_{C}}[\right.\right.\right.$. It might be noted that the original grid $\mathbf{x}$ can be independently separated into the cylindrical and spherical components as $\mathbf{x}_{C y l}(i, m)$ (with $i \in\left[0 ; n_{s}\left[\right.\right.$ ) and $\mathbf{x}_{S p h}(i, m)$ (with $i \in$ $\left[n_{s} ; n_{s}+n_{\varphi}[)\right.$, respectively.

2) Hard Regularization: Due to the curvilinear nature of the proposed model, a strategy to prevent folding of surface regions is required. Indeed, at these position, points in the 3D Cartesian space can be described by multiple coordinates $\mathbf{x}_{C y l}(i, m)$ in the cylindrical space, which could result in intersections or folding portions along the final surface. Therefore, a hard regularization term is applied to preserve the surface shape (Fig. 2b). This term relies on a map, $\mathbf{r}^{\text {Hard }}$ (defined in the cylindrical space only), representing the nearest possible intersection or folding radii position (described as $r_{i, m}^{\text {Hard }}$ ) for each specific coordinate pair $\left(s_{i}, \theta_{m}\right)$. In detail, for each $\left(s_{i}, \theta_{m}\right)$ (green arrows in Fig. 2a), the hard limit is computed by intersecting a line (denoted as a set of points $\left\{\left[r^{L}, s_{i}, \theta_{m}\right], \forall r^{L} \in \mathbb{R}^{+}\right\}$) with a set of planes representing the remaining $s$ levels of the cylindrical model (exemplified as dark lines in Fig. $2 b$ and expressed as $\left\{\left[r^{P}, s_{j, j \neq i}, \theta^{P}\right], \forall\left\{r^{P} \in \mathbb{R}^{+}, \theta^{P}[0 ; 2 \pi[\}\}\right.\right.$, with $\left.j \in\left\{[i-v ; i+v] \cap\left[0 ; n_{s}\right]\right\}\right)$. Please note that by intersecting a specific line with a particular $s$ level, one obtains a local estimation of the maximum radii value where no folding regions are found. Moreover, since multiple intersection positions can occur along a specific line (particularly, if considered the intersection between the line and all the remaining $s$ levels), the final hard boundary position $\left[r_{i, m}^{\text {Hard }}, s_{i}, \theta_{m}\right]$ is defined as the intersection position with minimal radii (red dots in Fig. 2b). Finally, to limit the influence of too far planes on a particular position, which would drastically restrict the model and make the method computationally demanding, only the nearest $v$ planes are considered for each line (Fig. 2b). Additionally, in order to reduce the search region and assuming a given maximum size for the target object, a maximum radii $\beta$ was considered.

In order to regularize the surface model throughout the evolution process, we included the following feature:

$$
\bar{g}^{\operatorname{Reg}}\left(\mathbf{x}^{*}\right)=-H\left(\mathbf{x}^{*}-\mathbf{r}^{H a r d}\right)
$$




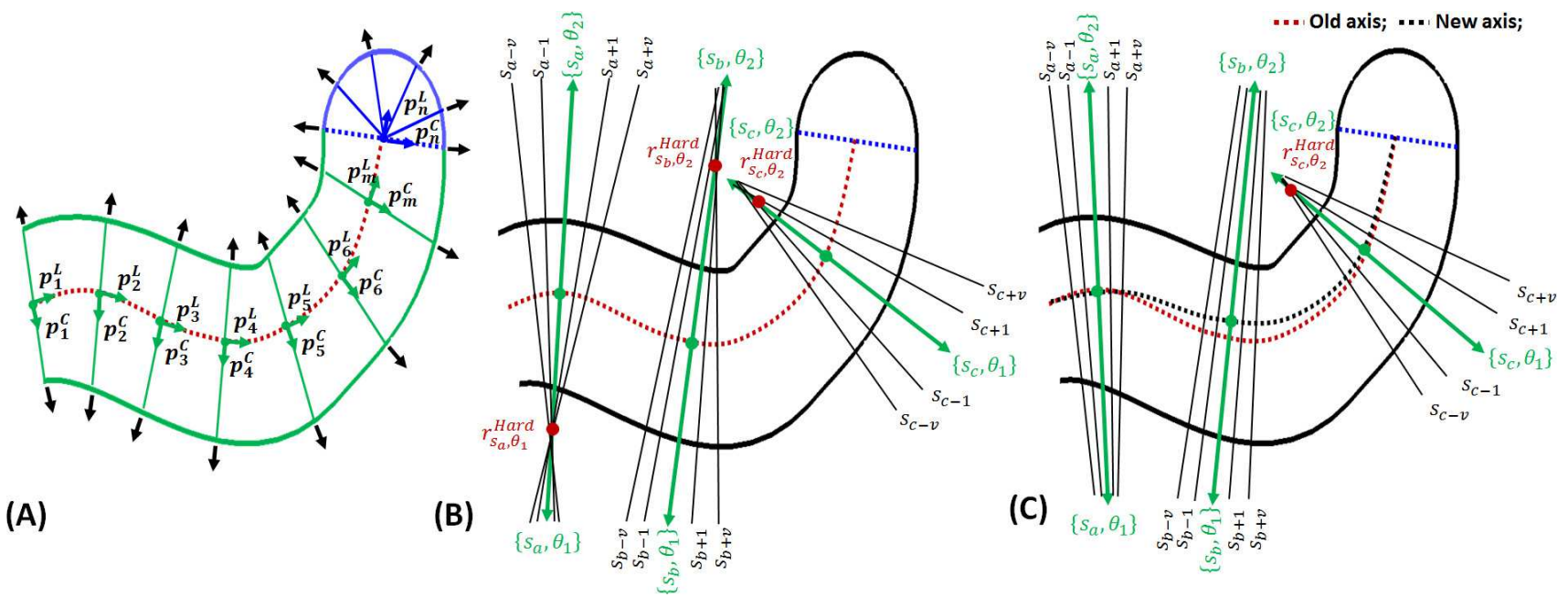

Fig. 2. (A) Schematic of the curvilinear blind-ended model; (B) Hard regularization strategy; (C) Axis recenter approach.

with $H$ representing a Heaviside operator. As such, at possible folding positions (i.e., $\mathbf{x}^{*}-\mathbf{r}^{\text {Hard }}>0$ ), high penalization is locally applied at the surface, preventing therefore its growth and ultimately avoiding surface folding. In the opposite case (i.e., $\mathbf{x}^{*}-\mathbf{r}^{\text {Hard }}<0$ ), no regularization is applied. Since this regularization term is only locally applied during a few iterations to keep the LAA shape coherent (presenting a value of zero in the remaining situations and not influencing the models optimization), no specific tuning of the hyperparameter $\alpha$ was performed. Therefore, $\alpha=1$ was used in our experiments.

3) Axis Recenter: Since the proposed model is optimized based on an initial fixed axis, a highly curved or complex surface not representing the particularities of the patient's anatomy is expected after a few iterations, requiring therefore multiple hard boundaries to prevent folding regions. Thus, in order to minimize the influence of the hard regularization term throughout the optimization, a strategy to correct a highly curved axis is applied. By correcting these points of the axis (Fig. 2c), not only the influence of the hard boundaries on the final result is reduced, but also increase the potentialities of the model to capture more particularities of the anatomy. Specifically, for each iteration, points on the curved axis with high curvature are identified as regions with a hard boundary radii lower than the pre-defined maximum value $\beta$. Then, a novel axis (Fig. 2c) is estimated by fitting a spline to all other points of the original axis. Note that the extreme positions of the centerline are always kept during this process, working as anchor positions and allowing a reconstruction of a novel axis centered on the target anatomy. Finally, taking into consideration the novel axis, the entire surface (i.e., cylindrical and half sphere) is resampled accordingly.

\section{Framework Overview}

Taking into account the previous description of the curvilinear blind-ended model, an explanation of the LAA segmentation methodology is now presented (Fig. 3). A constant model is initialized, by manually defining a centerline using three or more sequential clicks along the LAA. The different clicks are provided in the $3 \mathrm{D}$ space, by freely navigating through the volume using three orthogonal planes. Two evolution stages are subsequently applied: 1) a fast evolution based on a BEASthreshold approach to capture the global shape of the LAA; and 2) refinement to the patient anatomy by applying a BEASsegmentation technique with regional/local energies.

1) Fast contour growing: Due to the sensitivity of modelbased segmentation approaches to the initialization, a strategy to compensate for sub-optimal initializations is presented. The current method uses global terms and large evolution steps to quickly evolve the LAA model, allowing to recover the global and non-refined LAA shape. No energies are used to control this stage, requiring simple decision strategies (namely, the number of iterations) to stop the process.

The global model is evolved using two independent terms: 1) direct image content through a fixed threshold value, and 2) a global edge map estimated using phase-based filters [26]. Both terms are computed based on a set of image profiles $P$ defined radially with respect to the centerline (yellow arrows in Fig. 4a). Regarding the first term, the threshold is computed assuming a Gaussian mixture model with 3 independent regions (i.e. lumen, LAA trabeculae and cardiac wall). Then, an Expectation-Maximization algorithm is applied to estimate the optimal location of each Gaussian, and the mean value of the second Gaussian model used as threshold $(t h)$. In its turn, the edge transitions (for each $P$ ) are estimated by evaluating the even and odd responses of each image profile by applying Riesz filters [27] and a band-pass zero-DC filter (which attenuates undesirable frequencies), followed by the searching of asymmetry positions in the image's spectrum (Fig. 4b). This method was selected due to its theoretical intensityinvariance, making it suitable for the low-contrast and noisy TEE images [28], [29]. In order to increase the method's robustness to image noise (particularly at the lumen), the signed version (focusing on dark-to-bright transitions) recently proposed by [30] is applied in the current study to obtain a rough estimation of the LAA shape. Moreover, for each $P$, only the position with maximum asymmetry was considered as a valid edge (Fig. 4c). Please note that, by combining both edge-based and image-based terms, the method robustness is notably increased. Indeed, the edge term constrains the model 

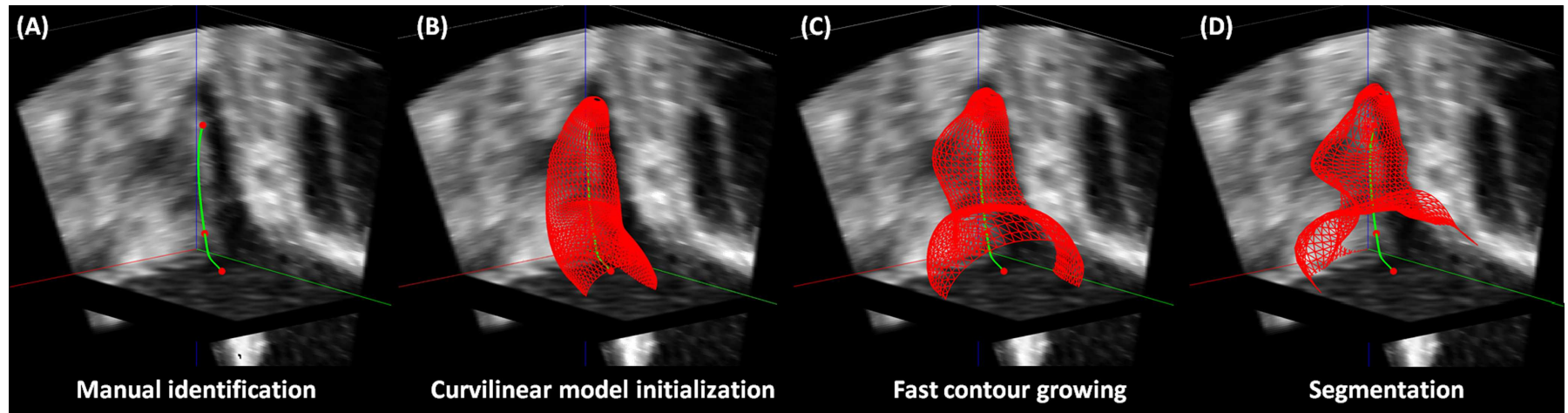

Fig. 3. Overview of the proposed LAA segmentation method.
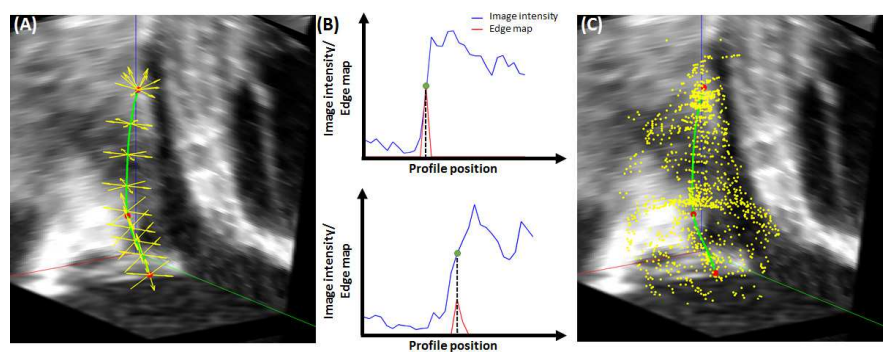

Fig. 4. Fast contour initialization strategy: (A) Image profiles (yellow arrows) are used to estimate the edge positions and the threshold value; (B) Edge estimation through a phase-based technique and (C) resulting edge positions.

evolution compensating for sub-optimal threshold values and making the strategy less sensitive to the number of iterations used. Moreover, due to the signed strategy, image noise at the lumen is easily surpassed. Finally, the combination of both terms improved the method's performance to distinguish between LAA trabeculae and wall.

The current methodology was embedded into the segmentation technique, by modifying Equation 5 as:

$$
\begin{gathered}
\bar{g}^{I M}\left(\mathbf{x}^{*}\right)=\bar{g}^{T h}\left(\mathbf{x}^{*}\right)+\bar{g}^{E d g e}\left(\mathbf{x}^{*}\right), \\
\bar{g}^{T h}\left(\mathbf{x}^{*}\right)= \begin{cases}1 & \text { if } \bar{I}\left(\mathbf{x}^{*}\right) \leq t h \\
-1 & \text { otherwise },\end{cases} \\
\bar{g}^{E d g e}\left(\mathbf{x}^{*}\right)=H\left(\mathbf{r}^{E d g e}-\mathbf{x}^{*}\right)-H\left(\mathbf{x}^{*}-\mathbf{r}^{E d g e}\right),
\end{gathered}
$$

with $\mathbf{r}^{E d g e}$ being a map representing the edge radii $\left(r_{i, m}^{E d g e}\right)$ for each image profile $P$ (described as a function of $p_{L_{i}}$ and $\left.p_{C_{m}}\right), \bar{I}\left(\mathbf{x}^{*}\right)$ is the image value at position $\mathbf{x}=\left\{x_{1}=\right.$ $\left.\psi\left(\mathbf{x}^{*}\right), x_{2}, \ldots, x_{n}\right\}$. Of note, the term $\bar{g}^{E d g e}$ is always attracting the contour to the nearest edge, pushing it outward and inward when the contour is inside (i.e. $\mathbf{x}^{*}-\mathbf{r}^{E d g e}<0$ ) or outside of the LAA blood pool (i.e. $\mathbf{x}^{*}-\mathbf{r}^{E d g e}>0$ ), respectively. It might be noted that, since both terms use a similar range (i.e. -1 or 1 ), no weight hyperparameter is required in equation 10 to balance the influence of the different feature maps.

2) Segmentation: The rough model is refined to the patient anatomy using a segmentation process with regional-based energies. The optimization process is guided by a local Yezzi energy [31], which estimates the optimal contour's position as the maximum contrast point between inner and outer regions. Moreover, since the LAA walls are brighter than the lumen, a signed version of this energy is used, explicitly embedding a specific representation of the target transition (i.e. darkto-bright) into the functional energy, improving therefore its robustness to noise and image artifacts.

This energy is integrated into the segmentation model by considering the following image criteria at Equation 2:

$$
F(\mathbf{y})=\omega_{i n} \cdot u_{x}-v_{x},
$$

and using its derivative to identify the local minima, through:

$$
\bar{g}^{I M}\left(\mathbf{x}^{*}\right)=\omega_{\text {in }} \frac{\bar{I}\left(\mathbf{x}^{*}\right)-u_{x}}{A_{u}}+\frac{\bar{I}\left(\mathbf{x}^{*}\right)-v_{x}}{A_{v}},
$$

where $u_{x}$ and $v_{x}$ are the mean intensities inside and outside of the evolving interface at point $\mathbf{x}$, calculated using mask $B . \omega_{i n}$ is a scalar weight applied to the inner region and $A_{u}$ and $A_{v}$ represent the area of the inner and outer region, respectively. It might be noted that due to presence of trabeculae at the LAA body (being darker than the wall and brighter than the lumen), a correct setting of $\omega_{i n}$ is required to intrinsically modify the equilibrium position between both inner and outer regions [31]. Moreover, due to the high performance typically found by the signed local Yezzi in ultrasound imaging, and contrary to section II-C1, no edge term is now applied.

\section{Implementation Details}

In order to increase the method's robustness to the initialization and to improve its performance at the initial portion of the LAA (i.e., interface between left atrium and LAA), a sequential segmentation scheme (section II-C2) is applied, namely: 1) initial estimation of the optimal contour position using a region $B$ with large profiles ( $\rho \simeq 6 \mathrm{~mm}$ ); and 2) result refinement using narrowing regions $B$ with more regional profiles $(\rho \simeq 2 \mathrm{~mm})$. Note that, since the walls around the LAA are typically thicker, the application of the current double-stage strategy improves the method's performance to identify the optimal boundary, without drastically increasing the risk of segmenting an outer structure.

A total of $40 \times 48$ points were used to represent the surface grid (i.e., $n_{s}=32, n_{\varphi}=8$ and $n_{\theta}=48$ ), with $h=2$ (for both coordinates $p_{L}$ and $p_{C}$ ). Moreover, $\omega_{i n}$ was set to 0.7 for all the experiments. The feature map presented in equation 5 is post-processed to prevent too large and local evolution steps caused by noisy regions or artifacts during the iterative process and to increase the stability of the segmentation 
process. Specifically, when the maximum value of the map (in absolute) is higher than 1, the entire map is normalized (keeping it in the range of $[-1 ; 1]$ ), dividing it by the maximum value. No post-processing is applied in the opposite situation (i.e. maximum value lower than 1). The fast initialization, based on BEAS-threshold and an edge term, was applied throughout 20 iterations and each profile $P$ had a length of $\sim 7 \mathrm{~mm}$. For the phase-based edge term, a band-pass zero-DC Difference-of-Gaussians filter was used. The two Gaussians were set with a sigma of approximately $2.3 \mathrm{~mm}$ and $3.2 \mathrm{~mm}$, respectively. Finally, regarding the hard regularization term, the maximum search region $(\beta)$ was set to $20 \mathrm{~mm}$ with a total of 5 neighboring planes $(v)$. In Section III-C, the influence of the selected terms and parameters is addressed and the influence of their variation explored.

\section{EXPERIMENTS}

Three different experiments were set to evaluate the performance of the method: 1) influence of the different parameters on the final method's accuracy; 2) evaluation of the segmentation error against manual analysis; and 3) differences between automated and manual analysis for the estimation of the clinical measurements for the planning of a LAA occlusion.

\section{A. Data Description}

A total of 20 TEE datasets were retrospectively selected from clinical practice, including patients with suspicion of pathology but with normal anatomy and abnormal cases (mainly at the left atria). Due to the shape variability typically found at the LAA, one expert classified each case based on [32], obtaining the following representation: chicken wings $45 \%$ (9 patients); windsock - 20\% (4 patients); cauliflower $20 \%$ (4 cases); and cactus - $15 \%$ ( 3 cases).

The acquisitions were performed using a 3D TEE probe with different ultrasound scanners, namely: GE Vivid E95 (GE Vingmed, Horten, Norway), Philips IE33 (Philips Ultrasound, Bothell, USA) and Philips Epiq 7. No modification to the normal clinical routine was required throughout the dataset acquisition. Specifically, an electrocardiogram (ECG)-gated acquisition was performed in mid-esophageal position using zoom mode and acquiring the LAA body, part of the mitral valve, left atrium and pulmonary veins [32]. The resulting image presented a resolution and size that varied from 0.20 to $0.45 \mathrm{~mm}$ and $126 \times 122 \times 117$ to $357 \times 413 \times 208$ voxels, respectively. The datasets were initially stored in a raw-data form and subsequently exported to a workstation with EchoPac (GE HealthCare, Horten, Norway) or QLab (Philips Ultrasound, Bothell, USA). Then, each case was anonymized, exported into an externally-readable format and converted to an isotropic voxel spacing. The entire dataset was constructed using the resources of the University Hospital Leuven with approval of the Ethics Committee (S59406).

\section{B. Ground Truth Generation}

Two observers manually delineated the 3D surface of the LAA for each patient, allowing the evaluation of the interobserver variability. The delineation was performed using a custom non-commercial software, Speqle3D [33], using the following strategy: 1) manual definition of the LAA centerline by providing three clicks along the anatomy; 2) 2D delineation of multiple LAA short-axis (SAx) views (30-40), defined perpendicular to the centerline; 3) 2D delineation of multiple LAA long-axis (LAx) views (2-5); and 4) 3D surface reconstruction using the obtained points along the different SAx and LAx views. Moreover, one of the observers repeated the analysis two weeks later, allowing the assessment of the intra-observer variability. In order to assess the influence of the initialization on the final result, each observer performed the analysis using independent LAA centerlines (henceforward referred as "Different axis" situation). Later, one observer repeated the segmentation process using the centerline defined by the other observer (termed as "Same axis" scenario).

Next, each observer manually extracted the relevant clinical measurements (defined as Obs1 and Obs2), namely: diameter of the ostium and landing zone and length of LAA, as described in [2], [8], [12]. Both observers performed this task independently, allowing the measurement of the reproducibility between them. For that, the user freely navigated along the 3D image using 3 orthogonal planes or resliced the data along the LAA centerline. The ostium and landing zone were defined by selecting the respective optimal image planes, and manually delineating their boundaries. Then, area-derived diameters were extracted for each case based on the $2 \mathrm{D}$ contours. The LAA depth was computed as the distance between the LAA tip (selected by the user in a LAx view) and the centroid of the landing zone. In order to assess the inter-observer variability caused by the $2 \mathrm{D}$ delineation process only, the second observer repeated the manual delineation at the ostium/landing zone defined by the first observer (henceforward referred as $O b s 2 R$ ). For the LAA depth, the second observer selected the LAA tip using the LAx view defined by the first observer.

\section{Parameter Tuning}

In the current experiment, an evaluation of the method's sensitivity to its parameters is performed. The following parameters were assessed: number of iterations used in the BEAS-threshold stage (section II-C1), the influence of the $\omega_{\text {in }}$ weight, the impact of the scaling term $(h)$, and the influence of the threshold value $(t h)$ in the final result. For each case, the nearest values to the selected one were evaluated. The th term was evaluated by summing/subtracting a fixed value $(5 \%, 10 \%, 15 \%$ and $20 \%$ of the intensity range of the full image) to the automatically estimated one. In order to prevent over-tuning, a total of 10 cases were randomly selected from the clinical database. Moreover, the automated method was initialized using the same centerline used throughout the manual segmentation by $O b s 1$.

The influence of each parameter was assessed in terms of the point-to-surface error ( $\mathrm{P} 2 \mathrm{~S}$ error), by comparing the automated segmentation result (for a specific parameter) against the manual result. An analysis of variance (ANOVA) was applied to each parameter to check for statistically significant differences $(p<0.05)$. Moreover, a two-tailed paired t-test $(p<0.05)$ was applied between each parameter and the optimal one. 

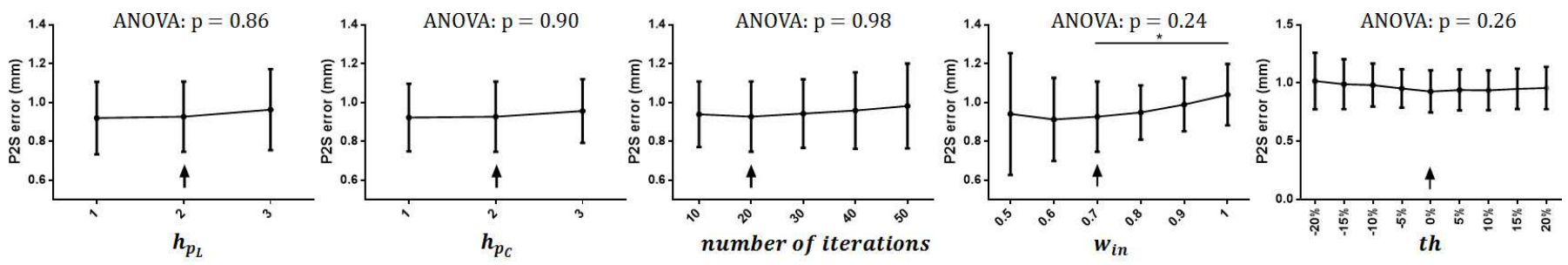

* $-\mathrm{p}<0.05$, two-tailed paired t-test between all pairs;

Fig. 5. Influence of the different segmentation parameters (scale in the longitudinal $h_{p_{L}}$ and circumferential $h_{p_{C}}$ directions, the number of iterations in section II-C1, the $\omega_{i n}$ ) and the threshold $t h$ value in the final accuracy of the proposed pipeline.

\section{Segmentation Accuracy}

The accuracy of the proposed segmentation technique is addressed throughout this experiment. In contrast to the previous experiment, the entire database (20 cases) is now used.

The difference (referred as Auto3D) between automated and manual analysis $(O b s 1)$, inter-observer and intra-observer variability were assessed in terms of P2S, Dice value and $95^{\text {th }}$ percentile of the Hausdorff distance (95HS). Similarly to section III-A, the automated method was evaluated in two scenarios: 1) considering the influence of the initialization, by initializing the automated method with a centerline different than the one used by the manual analysis; and 2) assuming an equal centerline for the automated and to the manual analysis. In order to check for statistically significant differences between each group (i.e. automated, inter- and intraobserver), a two-tailed paired t-test $(p<0.05)$ between each pair was computed. Moreover, in order to validate the proposed pipeline, the influence of each stage was assessed in terms of P2S error. Finally, the computational time of the automated method was compared with the time required by a manual analysis. All results were computed using MATLAB code (no parallelization) on a common personal laptop with Intel (R) i7 $\mathrm{CPU}$ at $2.8 \mathrm{GHz}$ and $16 \mathrm{~GB}$ of RAM.

\section{E. Clinical Measurements}

Similar to experiment III-D, the accuracy of the proposed automated method was compared with the manual analysis for the semi-automatic extraction of the relevant clinical measurements. Specifically for the automated method, the 3D LAA surface was clipped using the manually defined ostium and landing zone planes (independently selected by each observer), and the final clinical value estimated as an area-derived diameter (termed as Autol and Auto2 for Obs1 and $O b s 2$ levels, respectively). Regarding the automated LAA depth, it was computed as a distance between: 1) the centroid of the automated landing zone; and 2) the resulting position from the intersection between a line (manually defined by the observer by providing one click at the LAA tip and the centroid of its landing zone) and the 3D LAA surface.

The differences in clinical measurements between automated and manual results were evaluated in terms of bias (i.e., mean error of the differences), and limits of agreement (LOA, given as 1.96 times the standard deviation). The same analysis was performed to compare the difference between observers $(O b s 1-O b s 2)$ and even to evaluate the 2D delineation reproducibility $(O b s 1-O b s 2 R)$. For each pair, a two-tailed paired
TABLE I

SEGMENTATION ERRORS OF THE PROPOSED AUTOMATED TECHNIQUE

\begin{tabular}{cllc}
\hline \hline & P2S (mm) & Dice (\%) & 95th HS (mm) \\
\hline Auto3D & $0.88 \pm 0.20^{\mathrm{b}}$ & $82.5 \pm 3.6^{\mathrm{b}}$ & $2.49 \pm 0.73^{\mathrm{b}}$ \\
Inter-observer variability & $0.91 \pm 0.18^{\mathrm{b}}$ & $84.3 \pm 5.2^{\mathrm{b}}$ & $2.20 \pm 0.64^{\mathrm{b}}$ \\
Intra-observer variability & $0.53 \pm 0.15$ & $89.4 \pm 2.8$ & $1.42 \pm 0.45$ \\
\hline \hline
\end{tabular}

Both manual and autometed approaches were initialized with the same axis; ${ }^{a} p<0.05$, two tailed paired t-test against the inter-observer variability;

${ }^{b} p<0.05$, two tailed paired t-test against the intra-observer variability;
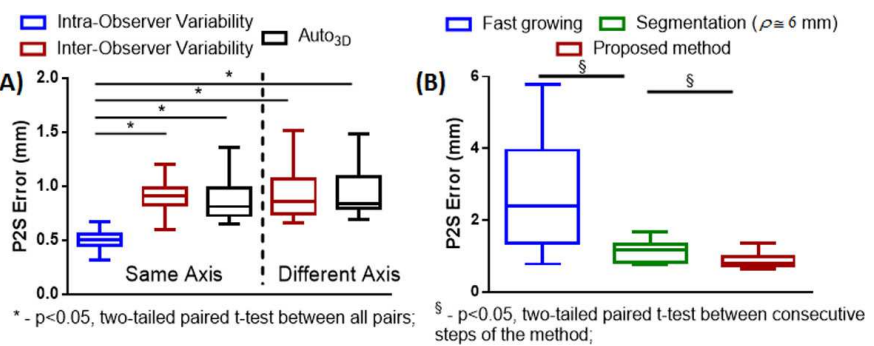

Fig. 6. (A) The accuracy of the proposed segmentation approach using the same and a different centerline; (B) P2S error throughout the different stages of the proposed method.

t-test and F-test were used to detect statistically significant differences $(p<0.05)$ in the biases and LOAs, respectively.

\section{RESUlts}

\section{A. Parameter Tuning}

Fig. 5 depicts the influence of the different parameters on the method's accuracy. The results corroborated the method's robustness, showing no statistically significant differences for the majority of the tested parameters (except for $\omega_{i n}$ ).

\section{B. Segmentation Accuracy}

Table I indicates the differences between the automated and manual analysis in terms of P2S error, Dice and 95HS error (at the "Same Axis" scenario). A similar performance was found when comparing the Auto3D error against the inter-observer variability, with statistically significant differences against the intra-observer variability. In terms of P2S error, a mean error lower than $1 \mathrm{~mm}$ was obtained for all the scenarios. The influence of the centerline axis was also addressed, showing no significant differences when modifying it (Fig. 6a). Fig. 6b also evaluates the method's performance at each step of the pipeline, showing a reduction (with statistically significant differences) between consecutive steps. Representative segmentation results are shown in Fig. 7. The proposed method 


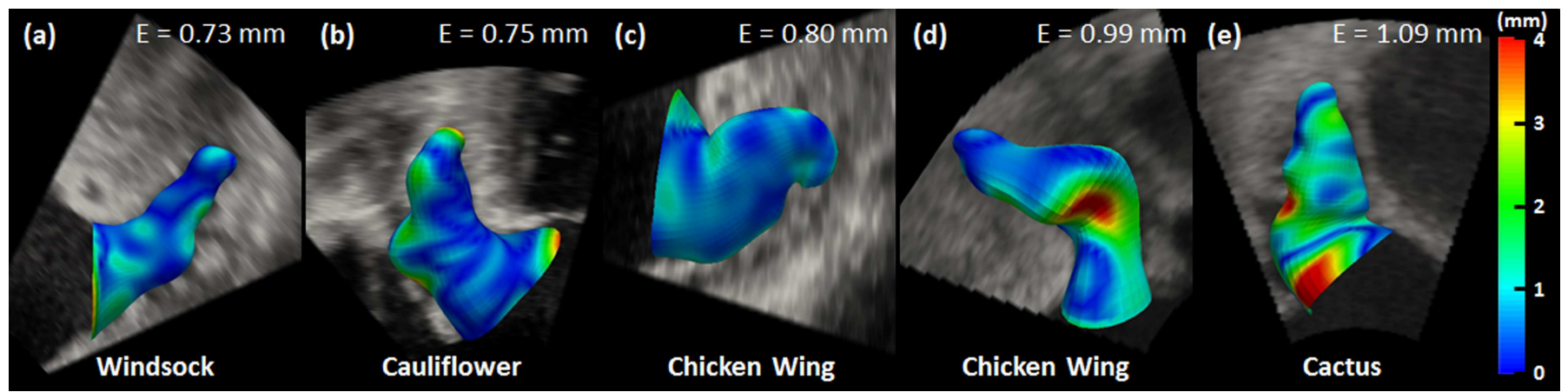

Fig. 7. Semi-automatic segmentation results corresponding to the (a) $10^{t h}$, (b) $30^{t h}$, (c) $50^{t h}$, (d) $70^{t h}$ and (e) $90^{t h}$ percentile of the P2S error.

required $14.0 \pm 4.8$ seconds (excluding initial centerline definition, which took $\sim 45$ seconds per case), while the manual analysis (i.e. 3D delineation) took $\sim 40$ minutes per case.

\section{Clinical Measurements}

Table II presents the differences between automated and manual analysis for each of the relevant clinical measurements. It might be noted that a centroid error (computed assuming the 2D contours) of $1.55 \pm 1.29 \mid 1.21 \pm 0.70 \mathrm{~mm}$ and a difference in terms of plane orientation of $10.15 \pm 5.05^{\circ} \mid 8.04 \pm 4.27^{\circ}$ were found between both observers for the ostium|landing zone planes, respectively. Globally, a similar performance was found between automated and manual approaches for the ostium, but with statistically significant differences at the landing zone and LAA depth. The worst performance in terms of automated and even inter-observer variability was found for the LAA depth. Low biases were found for the majority of the comparisons, presenting only statistically significant differences on Autol-Obsl at the landing zone and for the inter-observer variability for the LAA depth. Interestingly, the automated analysis versus a manual one always obtained narrower LOAs compared to the differences between observers. A second study was conducted where only the influence of the 2D delineation stage was evaluated (Table III). High similarity (in terms of bias and LOAs) was obtained between the automated method and the observer variability for all the measurements. Finally, Fig. 8 shows manual tracings and automated segmentation results at the ostium and landing zone. All tracings were contoured at the same SAx plane, showing accurate delineations for all the levels. Of note, the manual analysis took approximately 3 minutes (identification of the 3 measurements) per patient.

\section{DISCUSSION}

In this article, a novel semi-automatic methodology for LAA segmentation of peri-procedural data is proposed. The proposed method is initialized by a manually defined centerline along the LAA, which is subsequently corrected throughout the segmentation process, minimizing the influence of the observer's input and experience. Then, global and regional-based stages are applied to roughly estimate the LAA boundaries and to refine the contour to the real anatomy, respectively. Globally, the experimental findings corroborate the accuracy
TABLE II

AGREEMENT (IN MM) FOR AREA-DERIVED DIAMETER BETWEEN MANUAL ANALYSIS AND AUTOMATED DERIVED MEASUREMENTS

\begin{tabular}{ccccccccc}
\hline \hline & \multicolumn{2}{c}{ Ostium } & & \multicolumn{2}{c}{ Landing Zone } & & \multicolumn{2}{c}{ LAA depth } \\
\cline { 2 - 3 } & Bias & LOA & & Bias & LOA & & Bias & LOA \\
\hline Auto1-Obs1 & 0.38 & {$[-1.31 ; 2.07]$} & & $-0.42^{\mathrm{a}}$ & {$[-2.09 ; 1.24]^{\mathrm{c}}$} & & -0.35 & {$[-2.65 ; 1.85]$} \\
Auto2-Obs2 & 0.32 & {$[-1.45 ; 2.08]$} & & -0.05 & {$[-1.88 ; 1.78]$} & & $0.46^{\mathrm{b}}$ & {$[-1.55 ; 2.47]^{\mathrm{c}}$} \\
\hline Obs1-Obs2 & 0.02 & {$[-2.34 ; 2.39]$} & & 0.57 & {$[-2.13 ; 3.27]$} & & $0.80^{\mathrm{a}}$ & {$[-2.53 ; 4.12]$} \\
Auto1-Auto2 & 0.08 & {$[-1.73 ; 1.90]$} & & 0.20 & {$[-1.38 ; 1.78]^{\mathrm{c}}$} & & 0.01 & {$[-1.96 ; 2.00]^{\mathrm{c}}$} \\
\hline \hline
\end{tabular}

TABLE III

AGREEMENT (IN MM) BETWEEN AUTOMATED AND MANUAL ANALYSIS WHEN AT THE SAME LEVELS

\begin{tabular}{cccccccccc}
\hline \hline & \multicolumn{2}{c}{ Ostium } & & \multicolumn{2}{c}{ Landing Zone } & & \multicolumn{2}{c}{ LAA depth } \\
\cline { 2 - 3 } & Bias & LOA & & Bias & LOA & & Bias & LOA \\
\hline Auto1-Obs2R & 0.18 & {$[-1.63 ; 1.98]$} & & $0.52^{\mathrm{a}}[-1.22 ; 2.27]$ & & -0.05 & {$[-2.53 ; 2.42]$} \\
Obs1-Obs2R & -0.20 & {$[-1.80 ; 1.40]$} & & $0.95^{\mathrm{a}}[-1.13 ; 3.03]$ & & 0.30 & {$[-2.11 ; 2.72]$} \\
\hline
\end{tabular}

${ }^{a} p<0.05$, two-tailed paired t-test against $0 ;{ }^{b} p<0.05$, two-tailed F-test against Obs1-Obs2R; ${ }^{c} p<0.05$, two-tailed F-test against Obs1Obs2R; ${ }^{d} p<0.05$, two-tailed F-test against Auto1-Obs1; ${ }^{e} p<0.05$, two-tailed F-test against Auto1-Auto2; LOA - Limits of agreement (given as $\mu \pm 1.96 \sigma)$.

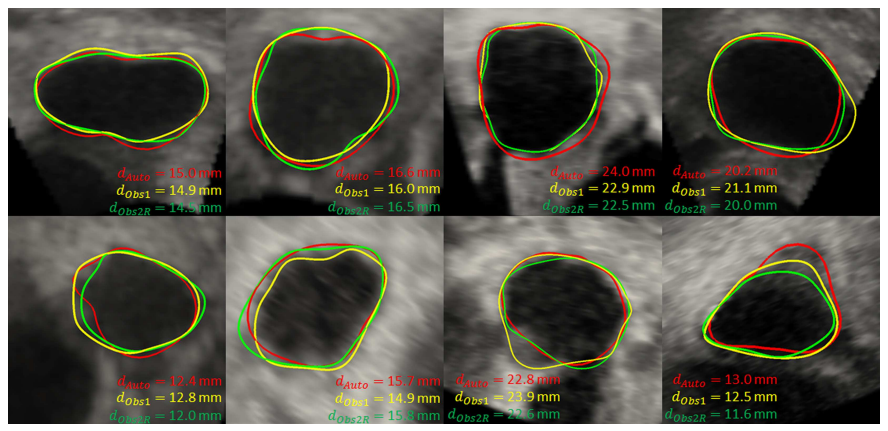

Fig. 8. Manual tracing (Obs1: yellow, Obs2R: green) and automated segmentation result (red) at ostium (1st line) and landing zone (2nd line) levels.

and feasibility of the proposed method, showing its potential for normal clinical practice, by generating a realistic $3 \mathrm{D}$ anatomical model that eases the interpretation of the TEE images. Moreover, it also simplifies the identification of the LAA shape and can even be used for an accurate planning of LAA occlusion intervention by semi-automatically extracting the relevant measurements for the selection of the optimal 
device. Finally, due to its low computational time, which is approximately 100 times faster than the manual segmentation, the potential and interest of the proposed methodology is enlarged. Additionally, thanks to the current method and its low computational cost (less than 20 seconds), 3D evaluation of the LAA anatomy throughout the peri-procedural stage (which typically requires several minutes) is now possible and feasible, adding relevant anatomical information and potentially improving the current interventional practice.

In contrast to other LAA segmentation techniques applied on CT images [19], [20], the proposed method, to the best of our knowledge, is the first directly applied on peri-procedural TEE data. Although previous studies compared both CT- and 3D TEE-based planning of LAA occlusion [12]-[14], [16], [17], [34], suggesting a superior performance of the first one [13]-[15], this is still sub-optimal due to the possible anatomical variations after the pre-operative acquisition and even the radiation exposure required [16]. Since one of the major limitations of the 3D TEE-based planning is the correct interpretation of the images, showing high variability between observers, automated tools to facilitate this task are of special interest [8]. Thus, by applying the proposed solution in 3DTEE data, an exact evaluation of the patient's anatomy is allowed, ultimately improving the current planning scenario. Of note, although the proposed method is described as the first automated approach for segmentation of LAA in 3D TEE, a previous work [8] applied interactive and time-demanding strategies, i.e. threshold selection followed by manual correction, to obtain the 3D surface.

The proposed segmentation strategy is performed based on a curvilinear blind-ended model embedded into the BEAS framework (Fig. 2 and 3). In contrast with previous BEAS techniques applied to different cardiac chambers using individual coordinate systems [24], [25], the current complex model was constructed by combining two independent spaces. In order to prevent any type of artifacts at the transition zone between both coordinate systems, both models are simultaneously optimized and combined into a unique grid of Bspline coefficients (see Fig. 2 and 7). Moreover, due to the curvilinear nature of the method, a regularization approach was required to control the model shape (preventing folding), which showed high performance in the current database (see supplementary material I). Although the current blind-ended model was proposed and validated to segment the LAA, it also shows potential for other situations, such as the left ventricle (LV) and LA. Indeed, recently, researchers [35] applied a similar concept (i.e., fusion of different coordinate systems) on an anatomical tracking of the LV.

In order to evaluate the proposed method, a comparison between the automated result and the traditional manual delineation strategy was initially performed. The values in Table I proved the high accuracy $(\sim 0.9 \mathrm{~mm})$ of the automated method, obtaining a performance similar to the one seen between observers (see also supplementary material II). This high performance is corroborated by Fig. 7. The highest errors were found at the opening region (transition between LA body and LAA) and the LAA tip. The first is easily explained by the axis correction strategy. Indeed, small modifications along the longitudinal direction of the initial axis will result in different opening planes, and therefore in large differences between surfaces. Additionally, at this plane, specific portions of the lumen-wall interface can be missed due to the limited field of the TEE acquisition, hampering the evolution of the proximal LAA model. The second is explained by the low contrast found at the LAA tip, as previously reported in [8]. Statistically significant differences were found when comparing the automated result with the intra-observer variability. This result demonstrated the high robustness of the manual delineation approach when performed by the same observer, showing a significant worst result when considering the interobserver variability. The automated method clearly improves this scenario, making the method less dependent on the user. Moreover, the automated method is much faster than manual analysis, making it an attractive solution for the clinical usage.

Regarding the influence of the different model parameters (Fig. 5), the results showed high robustness to their variation, obtaining non-statistically significant differences for the majority of the situations. Only a careful selection of the $\omega_{i n}$ weight is required to compensate the presence of trabeculae between the LAA blood pool and the wall. The influence of the manual initialization was also addressed, by evaluating the method's performance using a second independent axis (Fig. 6a). A slightly worst result was obtained when compared with the initial study, but with no statistically significant differences. It should be noticed that since the axis is constantly corrected throughout the proposed pipeline, the influence of sub-optimal initializations is minimized throughout the optimization process. The importance of each algorithm's step was also evaluated (Fig. 6b). A significant improvement of the method's accuracy was always observed between consecutive steps, validating the proposed method. In fact, and as previously demonstrated by other studies [25], model initialization using a fast growing approach (section II-C1) shows high feasibility and robustness. Here, it should be highlighted the high robustness of this fast growing strategy to the variation of the th, showing a similar performance (statistically not significant) even when modifying it using $20 \%$ of the full image intensity range (Fig. 5). Then, the rough shape (obtained during the fast growing stage) is locally adapted using a double segmentation stage, where the method starts by globally identifying the optimal boundary (using a large search window) and refining it through a small search window.

In addition to the previous validation, the performance of the proposed method in terms of semi-automatic extraction of the relevant clinical measurements for occluding device selection was evaluated. Table II proved the high performance of the automated method, obtaining narrow LOA for all the situations, even when compared with the inter-observer variability (with statistically significant differences for the landing zone and LAA depth). The advantages of the automated results are corroborated when considering the mean size of each clinical measurement (ostium - $20.2 \pm 5.2 \mathrm{~mm}$, landing zone $-17.1 \pm 4.5 \mathrm{~mm}$ and LAA depth $-20.5 \pm 7.5$ $\mathrm{mm}$, further details on supplementary material III). Moreover, when compared to previous studies focused on the LAA occlusion intervention, notably narrower LOAs were obtained 
[13], [16], [17]. Nevertheless, the majority of them focused on multi-modality validation studies, explaining these large differences. Specifically for the ostium and landing zone, the current results corroborated the advantage of the proposed approach, i.e., estimating the relevant clinical measurements based on a full 3D surface, when compared with the traditional practice, where an expert traces a given 2D plane without guaranteeing a 3D shape consistency. Indeed, this independent approach is prone to errors (due to image artifacts) and highly dependent on the observer. In contrast, by extracting a full 3D surface, a realistic and continuous model with a certain degree of smoothing is obtained, reducing the variability found when measuring the relevant indicator at nearby locations (i.e., different planes selected by different observers). In fact, similar observations were obtained by a previous study focused on a different cardiac structure [24].

Assessing each measurement individually, the lowest errors were found at the level of the ostium while the largest differences were registered for the LAA depth. This tendency is explained by the clear and well-contrasted image usually found at the ostium (first line of Fig. 8) and the difficulties to correctly visualize the LAA tip due to its anatomical position in relation to the probe tip [8], respectively. Interestingly, although constant differences in terms of delineation strategy were found between observers, an intermediate result was always obtained by the automated method. The influence of the $2 \mathrm{D}$ measurement extraction at the same plane was also assessed (Table III). A reduction of the inter-observer variability was seen for all measurements, but without statistically significant differences with respect to Table II. Remarkably, and in contrast to the previous observation, a high similarity is now found between the automated result (in both Tables II and III) and the inter-observer variability (Fig. 8). In fact, the proposed method achieved a similar performance when evaluated in both scenarios (i.e., measurement extracted at different levels/positions defined by different observers - Table II - and at the exact same level/position - Table III), corroborating the method's robustness and emphasizing its potential for normal clinical practice. Notwithstanding, statistically significant biases were again found at the landing zone level, again demonstrating the difficult analysis at this level.

Regarding the obtained 3D shapes (Fig. 7 and supplementary material I), high anatomical variability was found between patients. Note that the clinical database used presented representative cases of the different LAA types with a similar prevalence to previous studies [17], [32]. By evaluating the obtained 3D shape, correct classification (i.e., as chicken wings, cactus, windsock or cauliflower) is facilitated, since the main properties of each class are captured by the model. Indeed, unilobular and multi-lobular structures were possible to be segmented (supplementary material I), being the cases with higher prevalence [32]. Nevertheless, the final result is highly smoothed when compared with the real anatomy, failing to capture specific details [11]. Moreover, in particular situations, small bridges connecting consecutive, nearby and small lobes can occur, slightly modifying the LAA shape and being a drawback of this technique. Nevertheless, these small lobes tend to be less important for the intervention. Additionally, extreme, totally independent and large multi-lobular structures are not suitable to be processed by the current methodology. Notwithstanding, the authors would like to emphasize that even with the abovementioned slightly modified shapes, correct extraction of the relevant clinical measurements is still possible. Since the relevant measurements (namely, ostium and landing zone) are typically extracted at the proximal portion, which is usually tubular and free of large multi-lobes, the method's applicability in these situations is not limited, reinforcing again the added-value of the proposed technique.

\section{CONCLUSION}

The proposed semi-automatic method proved its potential for LAA segmentation, showing high accuracy, robustness and low computational time. Furthermore, by performing the planning of LAA occlusion intervention through the obtained 3D model, it is expected that accurate and more reproducible measurements will be obtained, corroborating the added-value of the proposed method for daily clinical practice.

\section{ACKNOWLEDGMENT}

The authors would like to acknowledge Olivier Bernard (CREATIS, University of Lyon, France) for his contribution in the initial implementation of the curvilinear BEAS model.

\section{REFERENCES}

[1] B. Meier, Y. Blaauw, A. A. Khattab, T. Lewalter, H. Sievert, C. Tondo, M. Glikson, D. Reviewers, G. Y. Lip, J. Lopez-Minguez et al., "EHRA/EAPCI expert consensus statement on catheter-based left atrial appendage occlusion," Europace, vol. 16, no. 10, pp. 1397-1416, 2014.

[2] N. C. Wunderlich, R. Beigel, M. J. Swaans, S. Y. Ho, and R. J. Siegel, "Percutaneous interventions for left atrial appendage exclusion: options, assessment, and imaging using 2D and 3D echocardiography," JACC: Cardiovascular Imaging, vol. 8, no. 4, pp. 472-488, 2015.

[3] J.-W. Park, A. Bethencourt, H. Sievert, G. Santoro, B. Meier, K. Walsh, J. R. Lopez-Minquez, D. Meerkin, M. Valdés, O. Ormerod et al., "Left atrial appendage closure with Amplatzer cardiac plug in atrial fibrillation: initial European experience," Catheterization and Cardiovascular Interventions, vol. 77, no. 5, pp. 700-706, 2011.

[4] D. S. Beutler, R. D. Gerkin, and A. I. Loli, "The morphology of left atrial appendage lobes: a novel characteristic naming scheme derived through three-dimensional cardiac computed tomography," World Journal of Cardiovascular Surgery, vol. 4, no. 03, p. 17, 2014.

[5] S. Murarka, M. Lazkani, S. Moualla, D. R. Verma, and A. Pershad, "Left atrial anatomy and patient-related factors associated with adverse outcomes with the watchman devicea real world experience," Journal of interventional cardiology, vol. 30, no. 2, pp. 163-169, 2017.

[6] V. Y. Reddy, S. K. Doshi, S. Kar, D. N. Gibson, M. J. Price, K. Huber, R. P. Horton, M. Buchbinder, P. Neuzil, N. T. Gordon et al., "5-Year outcomes after left atrial appendage closure: From the PREVAIL and PROTECT AF trials," Journal of the American College of Cardiology, p. 24357, 2017.

[7] P. Morais, J. L. Vilaça, J. Ector, J. D’hooge, and J. M. R. Tavares, "Novel solutions applied in transseptal puncture: a systematic review," Journal of Medical Devices, vol. 11, no. 1, p. 010801, 2017.

[8] H. Song, Q. Zhou, Q. Deng, J. Chen, L. Zhang, T. Tan, and R. Guo, "Morphologic assessment of the left atrial appendage in patients with atrial fibrillation by gray values-inverted volume-rendered imaging of three-dimensional transesophageal echocardiography: A comparative study with computed tomography," Journal of the American Society of Echocardiography, vol. 29, no. 11, pp. 1100-1108, 2016.

[9] P. L. Pellegrino, G. Fassini, M. Di Biase, and C. Tondo, "Left atrial appendage closure guided by 3D printed cardiac reconstruction: emerging directions and future trends," Journal of cardiovascular electrophysiology, vol. 27, no. 6, pp. 768-771, 2016. 
[10] L. Christiaens, N. Varroud-Vial, P. Ardilouze, S. Ragot, J. Mergy, B. Bonnet, D. Herpin, and J. Allal, "Real three-dimensional assessment of left atrial and left atrial appendage volumes by 64-slice spiral computed tomography in individuals with or without cardiovascular disease," International journal of cardiology, vol. 140, no. 2, pp. 189196, 2010.

[11] L. Di Biase, P. Santangeli, M. Anselmino, P. Mohanty, I. Salvetti, S. Gili, R. Horton, J. E. Sanchez, R. Bai, S. Mohanty et al., "Does the left atrial appendage morphology correlate with the risk of stroke in patients with atrial fibrillation?: results from a multicenter study," Journal of the American College of Cardiology, vol. 60, no. 6, pp. 531-538, 2012.

[12] D. D. Wang, M. Eng, D. Kupsky, E. Myers, M. Forbes, M. Rahman, M. Zaidan, S. Parikh, J. Wyman, M. Pantelic et al., "Application of 3-dimensional computed tomographic image guidance to WATCHMAN implantation and impact on early operator learning curve: single-center experience," JACC: Cardiovascular Interventions, vol. 9, no. 22, pp. 2329-2340, 2016.

[13] O. Goitein, N. Fink, I. Hay, E. Di Segni, V. Guetta, D. Goitein, Y. Brodov, E. Konen, and M. Glikson, "Cardiac CT angiography (CCTA) predicts left atrial appendage occluder device size and procedure outcome," The international journal of cardiovascular imaging, vol. 33, no. 5, pp. 739-747, 2017.

[14] A. Rajwani, A. J. Nelson, M. G. Shirazi, P. J. Disney, K. S. Teo, D. T. Wong, G. D. Young, and S. G. Worthley, "CT sizing for left atrial appendage closure is associated with favourable outcomes for procedural safety," European Heart Journal-Cardiovascular Imaging, vol. 18, no. 12, pp. 1361-1368, 2016.

[15] J. R. López-Mínguez, R. González-Fernández, C. Fernández-Vegas, V. Millán-Nuñez, M. E. Fuentes-Cañamero, J. M. Nogales-Asensio, J. Doncel-Vecino, M. Yuste Dominguez, L. Garcia Serrano, and D. Sanchez Quintana, "Comparison of imaging techniques to assess appendage anatomy and measurements for left atrial appendage closure device selection," J Invasive Cardiol, vol. 26, no. 9, pp. 462-7, 2014.

[16] G. Nucifora, F. F. Faletra, F. Regoli, E. Pasotti, G. Pedrazzini, T. Moccetti, and A. Auricchio, "Evaluation of the left atrial appendage with real-time 3-dimensional transesophageal echocardiography," Circulation: Cardiovascular Imaging, pp. CIRCIMAGING-111, 2011.

[17] W. Bai, Z. Chen, H. Tang, H. Wang, W. Cheng, and L. Rao, "Assessment of the left atrial appendage structure and morphology: comparison of real-time three-dimensional transesophageal echocardiography and computed tomography," The international journal of cardiovascular imaging, vol. 33, no. 5, pp. 623-633, 2017.

[18] P. Liu, R. Liu, Y. Zhang, Y. Liu, X. Tang, and Y. Cheng, "The value of $3 \mathrm{D}$ printing models of left atrial appendage using real-time 3D transesophageal echocardiographic data in left atrial appendage occlusion: applications toward an era of truly personalized medicine," Cardiology, vol. 135, no. 4, pp. 255-261, 2016.

[19] P. Grasland-Mongrain, J. Peters, and O. Ecabert, "Combination of shape-constrained and inflation deformable models with application to the segmentation of the left atrial appendage," in IEEE International Symposium on Biomedical Imaging: From Nano to Macro, 2010. IEEE, 2010, pp. 428-431.

[20] L. Wang, J. Feng, C. Jin, J. Lu, and J. Zhou, "Left atrial appendage segmentation based on ranking 2-D segmentation proposals," in International Workshop on Statistical Atlases and Computational Models of the Heart. Springer, 2016, pp. 21-29.

[21] C. Jin, J. Feng, L. Wang, J. Liu, H. Yu, J. Lu, and J. Zhou, "Left atrial appendage segmentation using fully convolutional neural networks and modified three-dimensional conditional random fields," IEEE Journal of Biomedical and Health Informatics, 2018.

[22] D. Barbosa, T. Dietenbeck, J. Schaerer, J. D'hooge, D. Friboulet, and O. Bernard, "B-spline explicit active surfaces: an efficient framework for real-time 3-D region-based segmentation," IEEE transactions on image processing, vol. 21, no. 1, pp. 241-251, 2012.

[23] S. Lankton and A. Tannenbaum, "Localizing region-based active contours," IEEE transactions on image processing, vol. 17, no. 11, pp. 2029-2039, 2008.

[24] S. Queirós, A. Papachristidis, D. Barbosa, K. C. Theodoropoulos, J. C. Fonseca, M. J. Monaghan, J. L. Vilaça, and J. D'hooge, “Aortic valve tract segmentation from 3D-TEE using shape-based B-spline Explicit Active Surfaces," IEEE transactions on medical imaging, vol. 35, no. 9, pp. 2015-2025, 2016.

[25] P. Morais, J. L. Vilaça, S. Queirós, F. Bourier, I. Deisenhofer, J. M. R. Tavares, and J. D'hooge, "A competitive strategy for atrial and aortic tract segmentation based on deformable models," Medical Image Analysis, vol. 42, pp. 102-116, 2017.
[26] A. Belaid, D. Boukerroui, Y. Maingourd, and J.-F. Lerallut, "Phasebased level set segmentation of ultrasound images," IEEE Transactions on Information Technology in Biomedicine, vol. 15, no. 1, pp. 138-147, 2011.

[27] M. Felsberg and G. Sommer, "The monogenic signal," IEEE Transactions on Signal Processing, vol. 49, no. 12, pp. 3136-3144, 2001.

[28] V. Grau, H. Becher, and J. A. Noble, "Registration of multiview realtime 3-D echocardiographic sequences," IEEE transactions on medical imaging, vol. 26, no. 9, pp. 1154-1165, 2007.

[29] K. Rajpoot, V. Grau, and J. A. Noble, "Local-phase based 3d boundary detection using monogenic signal and its application to real-time 3D echocardiography images," in IEEE International Symposium on Biomedical Imaging: From Nano to Macro, 2009. ISBI'09. IEEE, 2009, pp. 783-786.

[30] H. R. Torres, "Kidney segmentation in 3D ultrasound images," Master Thesis. University of Minho., 2016.

[31] S. Queirós, D. Barbosa, B. Heyde, P. Morais, J. L. Vilaça, D. Friboulet, O. Bernard, and J. Dhooge, "Fast automatic myocardial segmentation in 4D cine CMR datasets," Medical image analysis, vol. 18, no. 7, pp. 1115-1131, 2014.

[32] R. Beigel, N. C. Wunderlich, S. Y. Ho, R. Arsanjani, and R. J. Siegel, "The left atrial appendage: anatomy, function, and noninvasive evaluation," JACC: Cardiovascular imaging, vol. 7, no. 12, pp. 12511265, 2014.

[33] S. Queirós, P. Morais, C. Dubois, J.-U. Voigt, W. Fehske, A. Kuhn, T. Achenbach, J. C. Fonseca, J. L. Vilaça, and J. D'hooge, "Validation of a novel software tool for automatic aortic annular sizing in threedimensional transesophageal echocardiographic images," Journal of the American Society of Echocardiography, vol. 31, no. 4, pp. 515-525, 2018.

[34] L. P. Budge, K. M. Shaffer, J. R. Moorman, D. E. Lake, J. D. Ferguson, and J. M. Mangrum, "Analysis of in vivo left atrial appendage morphology in patients with atrial fibrillation: a direct comparison of transesophageal echocardiography, planar cardiac CT, and segmented three-dimensional cardiac CT," Journal of interventional cardiac electrophysiology, vol. 23, no. 2, pp. 87-93, 2008.

[35] Y. Zhou, O. Bernard, E. Saloux, A. Manrique, P. Allain, S. MakramEbeid, and M. De Craene, "3D harmonic phase tracking with anatomical regularization," Medical image analysis, vol. 26, no. 1, pp. 70-81, 2015. 